Pathologe 2008 · [Suppl 2] 29:112-117 DOI 10.1007/s00292-008-1027-x

Online publiziert: 28. September 2008

(c) Springer Medizin Verlag 2008
R. Kandolf ${ }^{1}$ B. Bültmann ${ }^{2} \cdot$ K. Klingel ${ }^{1} \cdot$ C.-T. Bock ${ }^{1}$

${ }^{1}$ Abteilung Molekulare Pathologie, Universitätsklinikum Tübingen

${ }^{2}$ Abteilung Allgemeine Pathologie und Pathologische Anatomie, Institut für Pathologie, Universitätsklinikum Tübingen

\title{
Molekulare Mechanismen und Konsequenzen kardialer Virusinfektionen
}

Die inflammatorische Kardiomyopathie zählt entsprechend der WHO-Klassifikation zu den spezifischen Kardiomyopathien und beinhaltet die Myokarditis, die mit einer kardialen Dysfunktion einhergeht [20]. Aus kardiopathologischer Sicht versteht man unter einer Myokarditis eine Schädigung kardialer Myozyten mit reaktiver Infiltration des Herzmuskels durch Entzündungszellen mit konsekutiver interstitieller Remodellierung. Ausgenommen sind ischämische Läsionen [18]. Aufgrund der Etablierung von In-situ-Hybridisierungstechniken zum Nachweis einer Virusinfektion des Herzmuskels und detaillierten Untersuchungen zu viralen und wirtsspezifischen Pathogenitätsdeterminanten sind derzeit die pathogenetischen Prinzipien der Enterovirus-induzierten inflammatorischen Kardiomyopathie am besten verstanden, insbesondere hinsichtlich der ätiopathogenetischen Relevanz von Coxsackieviren der Gruppe B (CVB; $[11,12,14])$. Darüber hinaus wurde durch In-situ-Hybridisierungsstudien erstmalig gezeigt, dass Parvovirus B19 (B19) aufgrund der Infektion intrakardialer Endothelien mit einer reaktiven Schädigung von Myozyten und kardialer Dysfunktion einhergehen kann [3, 6, 14]. Für die kardiopathologische Diagnostik wurden hochsensitive PCR-Methoden für den Nachweis erregerspezifischer Nukleinsäuren in Endomyokardbiopsien entwickelt, die aufgrund ihrer Sensitivität unter Berücksichtigung der notwendigen Qualitätskriterien von zentraler Bedeutung sind $[14,16]$. Die kardiopathologischen
Untersuchungen erfolgen auf der Grundlage der Dallas-Kriterien für die Diagnose einer Myokarditis [1], die durch Einbezug immunhistologischer und molekularbiologischer Techniken zum Nachweis einer Infektion des Herzmuskels erweitert wurden. Die Richtlinien der Dallas-Kriterien erfordern, dass die kardiopathologische Diagnose ausschließlich auf der Grundlage morphologischer Befunde erfolgt. Die Interpretation der Befunde erfordert allerdings eine enge Zusammenarbeit mit der Klinik, insbesondere unter Berücksichtigung akuter, chronischer und akut rezidivierender Krankheitsverläufe.

\section{Spektrum kardialer Virusinfektionen}

PCR-Studien legen nahe, dass neben Enteroviren auch Infektionen mit Adenoviren, Parvovirus B19, humanem Herpesvirus 6, humanem Cytomegalievirus, Epstein-Barr-Virus, Herpes-simplex-Virus (HSV), humanem Immundefizienzvirus (HIV), Influenzaviren, Mumpsvirus, Rötelnvirus und Hepatitis-C-Virus (HCV) mit einer inflammatorischen Kardiomyopathie assoziiert sein können $[8,10$, 12, 14, 16, 18, 19]. Die • Abb. 1 zeigt das Spektrum viraler Infektionen, die in Tübingen mittels hochsensitiver „NestedPCR-Methoden" in Myokardproben von 3219 Patienten im Rahmen des etablierten kardiopathologischen Schwerpunktes bei klinisch vermuteter Myokarditis und dilatativer Kardiomyopathie auf der Grundlage der Dallas-Kriterien untersucht wurden: 1474 Patienten (46\%) erwiesen sich als viruspositiv. B19 ist mit $36,7 \%$ offensichtlich der häufigste potenziell kardiotrope Erreger, gefolgt von humanem Herpesvirus $6(20,1 \%)$ und Enteroviren $(7,9 \%)$. Hervorzuheben sind auch Doppelinfektionen mit B19 und HHV6 in $11,6 \%$ der untersuchten myokardialen Gewebeproben [10]. Die entsprechenden immunhistologischen Untersuchungen belegen, dass allerdings nur bei $62 \%$ der viruspositiven Endomyokardbiopsien eine pathologisch signifikante Entzündungsreaktion gesichert werden kann, die eine ätiopathogenetische Bedeutung der Infektion für die kardiale Beschwerdesymptomatik nahelegt. Bei immunhistologisch nicht nachweisbarer chronischer entzündlicher Begleitaktivität ( $38 \%$ der viruspositiven Patienten) ist aus infektiologischer Sicht definitionsgemäß von einer latenten Viruspersistenz ohne pathogenetische Bedeutung auszugehen. Allerdings können latente Infektionen insbesondere von DNA Viren wie B19 und HHV6 reaktivieren z. B. durch Koinfektionen, aber auch im Rahmen einer Herzinsuffizienz nichtviraler Ursache, wodurch rezidivierende Krankheitsverläufe sowie koinzidale Myokarditiden bei ätiologisch nicht primär viral bedingten Kardiomyopathien erklärt werden. Eine systemische Infektion als Beleg für eine akute oder kürzlich abgelaufene Infektion/Reaktivierung einer Infektion wird bei ca. 10\% der Patienten mit positivem Virusnachweis in der Endomyokardbiopsie beobachtet. 


\section{Molekulare Pathologie der Enterovirus-induzierten inflammatorischen Kardiomyopathie}

Humanpathogene Enteroviren, insbesondere Coxsackieviren der Gruppe B (CVB), sind ursächliche Erreger einer Myokarditis, Meningitis und Insulitis mit fraglicher Assoziation zum Typ-I-Diabetes [11]. Sie bilden kleine (28-30 $\mathrm{nm}$ ) ikosaedrische Partikel, die eine Positivstrang-RNA (ca. $7,4 \mathrm{~kb}$ ) enthalten. Voraussetzung für die Infektion von Myozyten ist die Bindung des Virus an zelluläre Rezeptorproteine. Hauptsächliches Rezeptorprotein ist ein 46-kDa-Transmembranprotein der Immunglobulin-Superfamilie mit Spezifität für Coxsackieviren der Gruppe B sowie für Adenovirus 2 und 5 (ADV2, $\mathrm{ADV}_{5}$ ), wobei dieses sog. „CAR-Protein“ (Coxsackie-Adenovirus-Rezeptorprotein), wie in $\bullet$ Abb. 2a gezeigt, präferenziell in den Glanzstreifen des menschlichen Herzens exprimiert wird [21]. Während der virämischen Phase erhält das Virus nach Transzytose des Endothels kleiner Kapillaren der Endstrombahn Zugang zu den Glanzstreifen des Myokards. Die spezifische Bindung des Virus an den CAR-Rezeptor induziert die rezeptorvermittelte Endozytose des Virus. Nach dem Eintritt in die Zelle wird das sog. „uncoating“ eingeleitet, durch das die virale RNA, die das infektiöse Prinzip darstellt, in das Zytoplasma zur Virusvermehrung entlassen wird. Im Anschluss an die akute Virusreplikation in hämatogen infizierten Myozyten (• Abb. 2b) kommt es zur Zell-zuZell-Ausbreitung der Infektion mit konsekutiver Zunahme der Organinfektion. Die reaktive akute Entzündung ist charakterisiert durch die Infiltration des Myokards mit CD68-positiven Makrophagen und „Natural-Killerzellen“ in einer ersten Welle der Entzündungsantwort (unspezifische Immunantwort), gefolgt von T-Helfer-Zellen (CD4) und zytotoxischen T-Lymphozyten (CD8) in einer zweiten Welle (spezifische Immunantwort). In dieser frühen Phase der reaktiven Entzündungsreaktion (• Abb. 2c) kommt es weiterhin zu einer Ausbreitung der Infektion, die zunehmend durch die Formierung der humoralen und zellulären Immunantwort eingedämmt wird. Die akute Entzündungs-

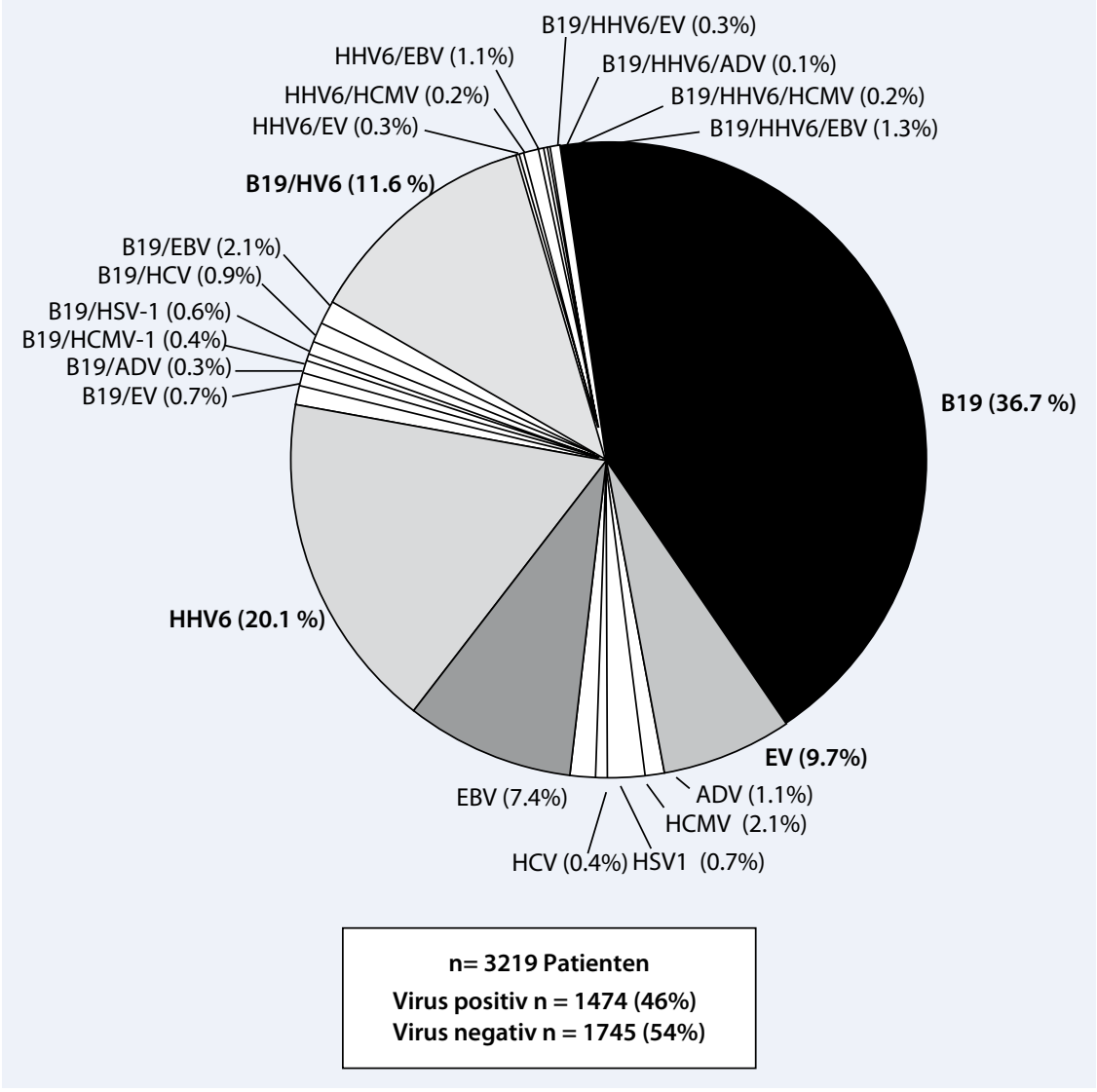

Abb. 1 A Spektrum viraler Infektionen in Myokardproben von 3219 Patienten mit kardialer Dysfunktion bei klinisch vermuteter Myokarditis und dilatativer Kardiomyopathie [10]. Im Vordergrund stehen Infektionen mit B19 (36,7\%), HHV6 (20,1\%) und EV (7,9\%). Inwieweit die zusätzlich dargestellten Doppel- und Dreifachinfektionen des Herzmuskels aufgrund viraler Interferenzen durch eine schlechtere Prognose charakterisiert sind, muss in klinischen Studien geklärt werden

reaktion (• Abb. 2d) erscheint trotz Expression kardiodepressiver Zytokine wie Interleukin-1- $\beta$ und TNF- $\alpha$ als protektiv, da es der zellulären Immunität in der Regel gelingt, infizierte myokardiale Zellen zu eliminieren. Dementsprechend heilen die meisten Myokarditiden aus. Im Rahmen der narbigen Defektheilung kann es jedoch zu einer bleibenden funktionellen Schädigung des Herzmuskels kommen.

In Abhängigkeit von der initialen Viruslast, der Formierung einer regulären Immunantwort sowie weiteren, bislang unbekannten genetischen Faktoren, kann sich das Virus der Überwachung durch das Immunsystem entziehen und hierdurch bei einem Teil der Patienten eine persistierende Infektion auslösen, die durch restringierte Virusreplikation charakterisiert ist. Das infektiöse Agens kann sich offensichtlich einer voll ausgeprägten Entzündungsreaktion entziehen und hierdurch über das Stadium der akuten Virusreplikation hinaus persistieren. Wichtig ist festzuhalten, dass es sich bei der chronischen Myokarditis nicht notwendigerweise um eine persistierende Virusinfektion handeln muss, da klinische Befunde auch eine postvirale Immunpathogenese der Myokardschädigung ohne Viruspersistenz belegen. Zudem kann eine persistierende enterovirale Infektion auch im Stadium einer chronischen inflammatorischen Kardiomyopathie noch spontan ausheilen.

Veränderte virale Replikationsstrategien, die Unfähigkeit von Effektoren der lokalen Immunität, persistent infizierte myokardiale Zellen zu eliminieren, sowie die Infektion von Immunzellen wurden als essenzielle Mechanismen der Induktion und Aufrechterhaltung einer chronischen virusassoziierten inflammatorischen Kardiomyopathie erkannt [12]. Dabei legen Befunde im transgenen Mausmodell durch herzmuskelspezifische Expression einer nichtlytischen replikativen $\mathrm{CVB}_{3}$ Variante nahe, dass auch die restringierte Virusreplikation mit dem Myozytenstoff- 
Pathologe 2008 · [Suppl 2] 29:112-117 DOI 10.1007/s00292-008-1027-x

(c) Springer Medizin Verlag 2008

\title{
R. Kandolf · B. Bültmann · K. Klingel · C.-T. Bock Molekulare Mechanismen und Konsequenzen kardialer Virusinfektionen
}

\begin{abstract}
Zusammenfassung
Ätiopathogenetische Hinweise für die Viruspathogenese der inflammatorischen Kardiomyopathie lassen sich mit molekularpathologischen Methoden ableiten. Neben Enteroviren (EV), insbesondere Coxsackieviren der Gruppe B (CVB), wurden Adenoviren, verschiedene Herpesviren und zunehmend Parvovirus B19 (B19) als potenziell kardiotrope Erreger identifiziert. Im Gegensatz zu den Enteroviren, die primär Myozyten infizieren, zeigt die B19-assoziierte inflammatorische Kardiomyopathie eine Infektion kleiner intramyokardialer Arteriolen und Venolen mit konsekutiver endothelialer Dysfunktion, akuter Mikrozirkulationsstörung und sekundärer Myozytenschädigung. Funktionelle Untersuchungen belegen eine Beteiligung von B19
\end{abstract}

an der intrazellulären Kalziumhomöostase durch die virale Phospholipase. Die B19-induzierte Caspase-Aktivierung kann zudem proinflammatorische Prozesse mit Dysregulation der STAT-Signalwege auslösen. Die Diagnose einer inflammatorischen Kardiomyopathie gelingt zweifelsfrei aus der Endomyokardbiopsie, wobei aus kardiopathologischer Sicht eine pathogenetisch bedeutsame inflammatorische Infektion von einer harmlosen myokardialen Viruspersistenz ohne Entzündung zu unterscheiden ist.

Schlüsselwörter Infektionspathologie - Virusinfektionen . Kardiomyopathie $\cdot$ Myokarditis · Entzündung

\section{Molecular mechanisms and consequences of cardiac viral infections}

\begin{abstract}
Molecular biological methods have confirmed the pathogenetic role of enteroviruses, primarily coxsackieviruses of group $B$ (CVB), in the induction and maintenance of inflammatory cardiomyopathy. More recently, adenoviruses, various herpes viruses, and increasingly parvovirus B19 (B19) have been identified as potential cardiotropic agents. While cardiac myocytes are target cells for enterovirus and adenovirus infections with virus-induced cytolysis, B19-associated inflammatory cardiomyopathy is characterized by infection of intracardiac endothelial cells of small arterioles and veins, which may be associated with endothelial dysfunction, impairment of myocardial microcirculation,
\end{abstract}

wechsel interferiert und somit von pathogenetischer Bedeutung ist [24].

Auf molekularer Ebene ist bekannt, dass enterovirale Proteinasen durch proteolytische Spaltung zellulärer Zielstrukturen mit dem Myozytenstoffwechsel interferieren. Die präferenziellen Zielstrukturen der viralen Proteinase $2 \mathrm{~A}^{\text {pro }}$ sind nicht nur Initiationsfaktoren der zellulären Translation, sondern auch zytoskelettale Proteine wie das Dystrophin, wobei diese von Badorff et al. [2] erhobenen Befunde erstmals eine Verbindung der viralen Herzerkrankung mit den genetisch determinierten Kardiomyopathien aufzeigten. Eigene Untersuchungen belegen, dass die virale Proteinase ${ }_{3} \mathrm{C}^{\text {pro }}$ bereits $5^{-}$ $6 \mathrm{~h}$ nach der Infektion das p21ras-Guanosintriphosphatase-aktivierende Protein (RasGAP) proteolytisch spaltet, wodurch der p21ras-Signalweg aktiviert wird mit konsekutiver Induktion des ERK/MAPKSignalwegs [9]. Hierdurch kommt es zu einer mitogenen Stimulierung der terminal differenzierten Myozyten, wodurch sich das Virus offensichtlich Replikationsvorteile im Sinne einer gesteigerten Virulenz verschafft. Die Aktivierung von MAP-Kinasen resultiert aber auch in einer Mobilisierung des intrazellulären Kalziums und ist somit an der Nekrobiose infizierter Myozyten beteiligt.

Die aktuelle Forschung konzentriert sich auf die Definition und funktionelle Charakterisierung von infektiologisch relevanten Suszeptilitätsfaktoren, welche die Induktion und Aufrechterhaltung einer chronischen enteroviralen inflammatorischen Kardiomyopathie auf molekularer Ebene erklären könnten [14, 21]. Dabei zeichnet sich ab, dass die im suszeptiblen Wirt initial höhere Viruslast im Vergleich zum resistenten Wirt mit einer unterschiedlichen zellulären Genexpression (z. B. Interferon-assoziierte Gene) mit verzögerter Expression von Immunoproteasomen, viraler Antigenprozessierung und zellulärer Immunantwort einhergeht.

\section{Parvovirus-B19-assoziierte inflammatorische Kardiomyopathie}

Parvovirus B19 (B19) ist ein kleines (18$26 \mathrm{~nm}$ ), nicht umhülltes DNA-Virus mit einem 5,7-kb-Einzelstrang-DNA-Molekül 
Abb. $2 \gg$ Pathogenese der Coxsackievirus-Myokarditis. Nach Bindung von Coxsackieviren an den Coxsackievirus-Adenovirus-Rezeptor (CAR), der präferenziell in den Glanzstreifen des menschlichen Herzens exprimiert wird

(a, Fluoreszenzimmunhistochemie), kommt es nach rezeptorvermittelter Endozytose zur Virusreplikation im Myozyten, wie im autoptischen Herzen eines erwachsenen Patienten (b, radioaktive ISH) sowie eines Kleinkindes (c, radioaktive ISH) gezeigt. In der Regel gelingt es der zellulären Immunität, im Rahmen der akuten reaktiven Entzündungsreaktion (d, CD3+-T-Lymphozyten) die akute Virusreplikation zu terminieren, wobei die Letalität bei der Säuglingsmyokarditis nach wie vor $50 \%$ beträgt. (Mit freundlicher Genehmigung aus [13])

Abb. 3 Akute B19-assoziierte inflammatorische Kardiomyopathie mit dem Insitu-Hybridisierungsnachweis viraler Genome in endothelialen Zellen einer kleinen intramyokardialen Arteriole (a) sowie in kleinen postkapillären Venolen (b). c Die endotheliale Infektion geht einher mit einer Adhäsion und Penetration von $\mathrm{CD} 3$-positiven T-Lymphozyten (rot) sowie CD68-positiven Makrophagen (braun) mit sekundären nekrobiotischen Veränderungen von Myozyten. d Die prominente Expression von MHC-Klasse-II-Molekülen (HLA-II-DR-alphaKette) in professionell antigenpräsentierenden $\mathrm{Ma}-$ krophagen und dendritischen Zellen sowie im Endothel reflektiert die B19induzierte Entzündung des Herzens. (Mit freundlicher Genehmigung aus [10])
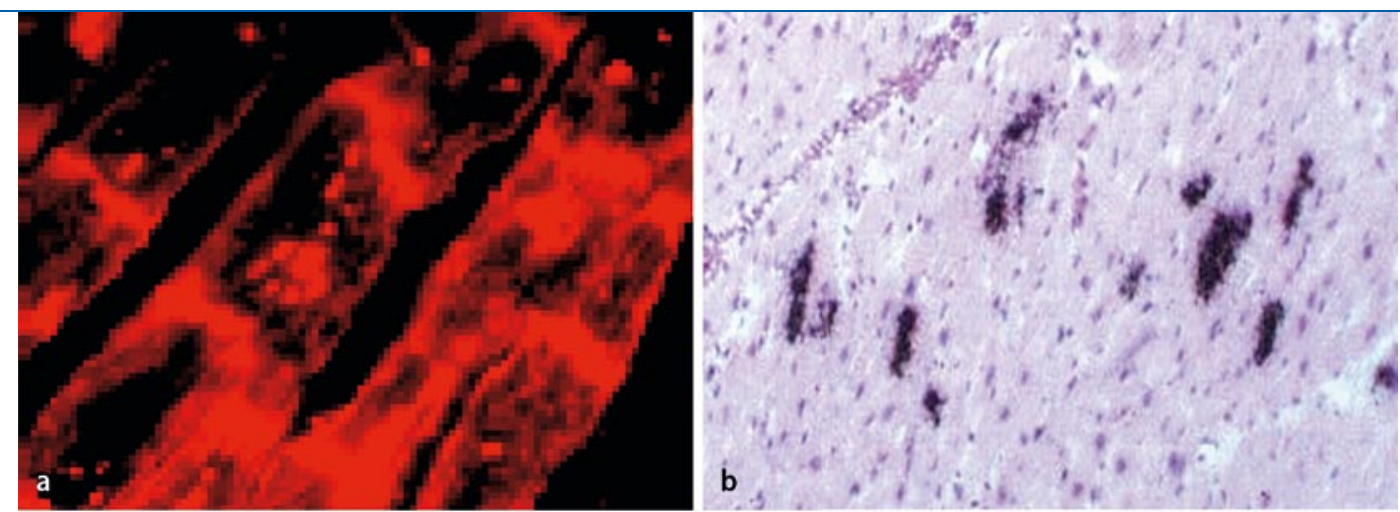

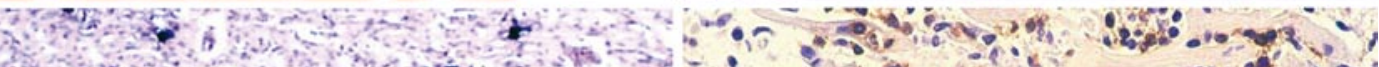

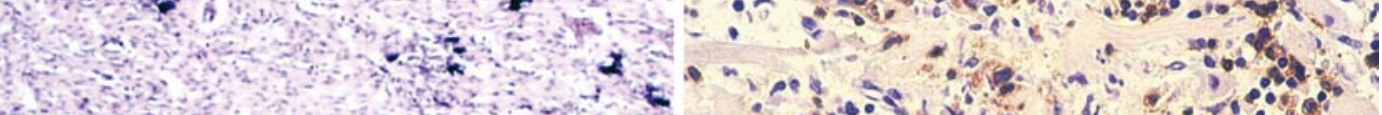
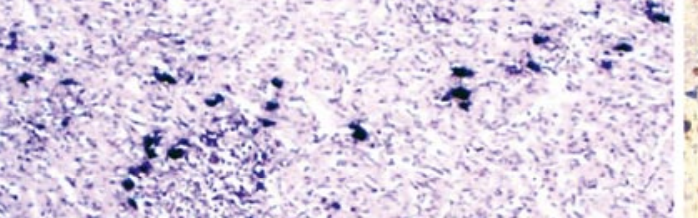

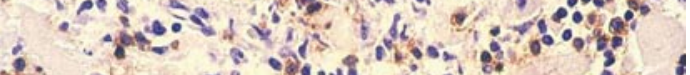

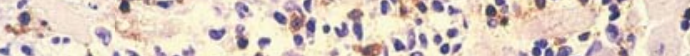

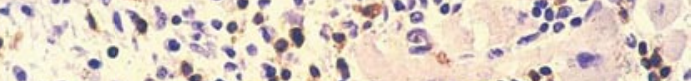
-

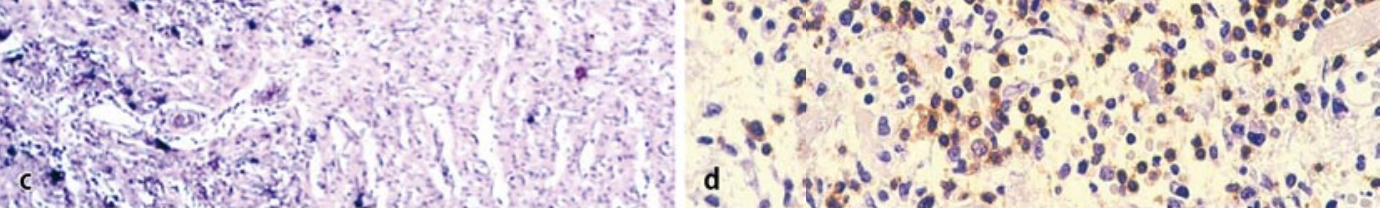
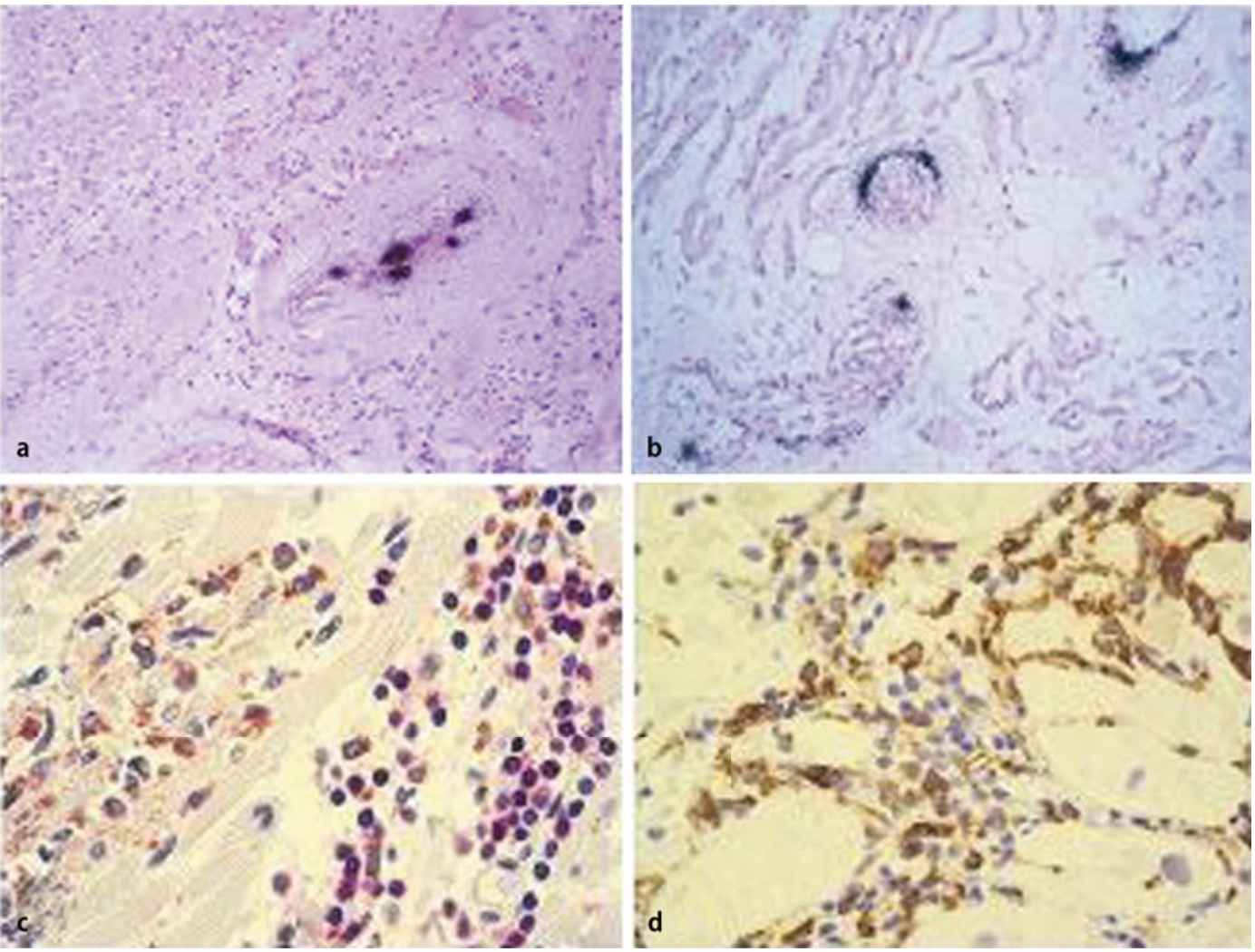


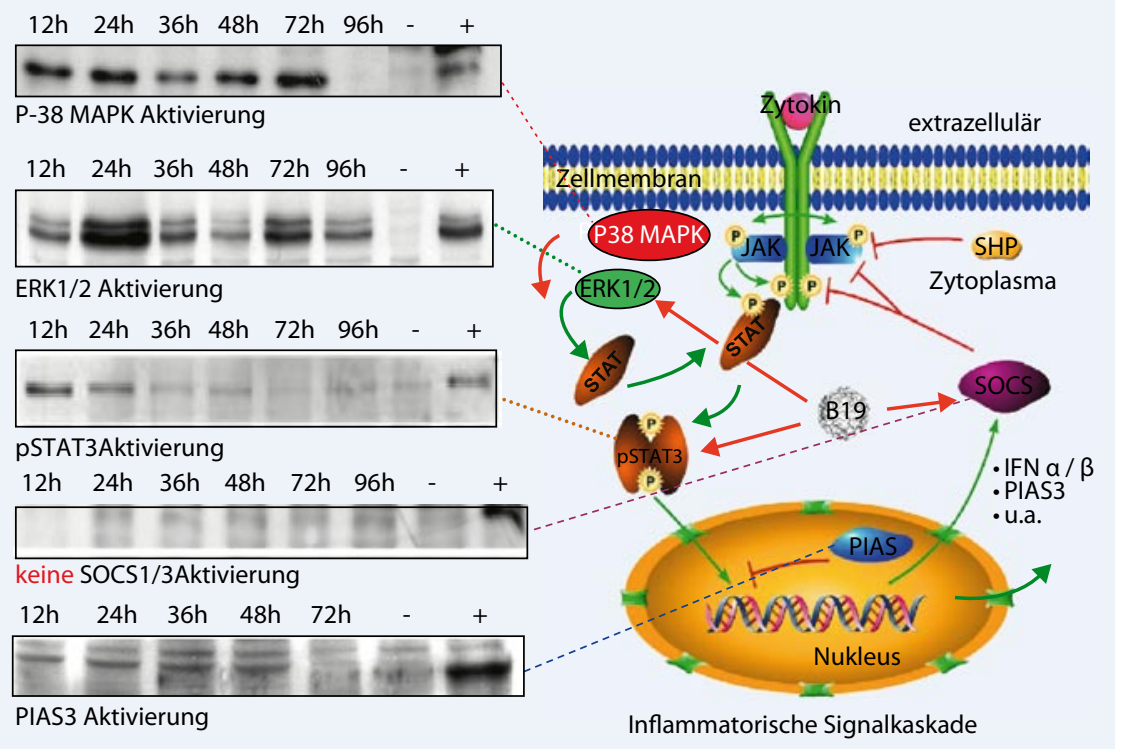

Abb. 4 A Western-Blot-Analysen zum Nachweis der Aktivierung der inflammatorischen Signaltransduktion durch die Parvovirus-B19-Infektion. P38-MAP-Kinase und P44/42-ERK-Kinase werden 72 bzW. $96 \mathrm{~h}$ nach B19-Infektion aktiviert. STAT3 zeigt eine Aktivierung bis lediglich $24 \mathrm{~h}$ nach B19-Infektion, wobei STAT3 durch seinen korrespondierenden Negativregulator PIAS3 nach $24 \mathrm{~h}$ herunterreguliert wird. Rechts schematisch dargestellt ist die inflammatorische Signaltransduktion des STAT/PIASSignalwegs mit Vernetzung zur MAPK/ERK-Signalkaskade

negativer Polarität und ursächlicher Erreger des Erythema infectiosum (Ringelröteln, „fifth disease“), des Hydrops fetalis und von transienten aplastischen Krisen bei angeborenen und erworbenen hämolytischen Anämien [3, 15]. PCR-Untersuchungen belegen die Assoziation von B19 mit einer Reihe von weiteren Erkrankungen wie Arthritis, Hepatitis, verschiedenen Vaskulitissyndromen und Myokarditis $[3,6,14,16]$. Als zellulärer Rezeptor für die Virusbindung wurde das Blutgruppen-P-Antigen identifiziert, ein Glykosphingolipid, das vornehmlich auf erythroiden Vorläuferzellen und Erythrozyten, aber auch auf Endothelzellen exprimiert wird [4, 15]. Als zellulärer Korezeptor für die erfolgreiche Virusaufnahme wurde $\alpha \mathrm{V} \beta \mathrm{I}$-Integrin beschrieben [23]. Infektiöse Viruspartikel können ausschließlich in proliferierenden, mitotisch aktiven Zellen gebildet werden [5].

Erste konkrete Hinweise für eine mögliche ätiopathogenetische Bedeutung einer B19-Infektion für die Entwicklung einer kardialen Dysfunktion ergaben sich aus Insitu-Hybridisierungsstudien mit der Identifikation intramyokardialer Endothelzellen, die offensichtlich spezifische Zielzellen für dieses Virus darstellen $[6,14]$. Die - Abb. 3 illustriert typische parvovirale Infektionsmuster des menschlichen Her- zens bei akuter B19-assoziierter inflammatorischer Kardiomyopathie. Sowohl kleine intramyokardiale Arteriolen ( $\bullet$ Abb. 3a) als auch postkapilläre Venolen und Venen (• Abb. 3b) sind durch eine hohe Viruslast des Endothels charakterisiert. Im Rahmen der endothelialen Infektion kommt es zu einer Expression von Adhäsionsmolekülen, insbesondere von E-Selektin, gefolgt von einer ausgeprägten intravaskulären Akkumulation, Margination, Adhäsion und Penetration von T-Lymphozyten und aktivierten Makrophagen, die das umgebende Myokard infiltrieren ( $\bullet$ Abb. 3c). Dies geht einher mit einer prominenten Expression von HLA-II-DR-Alpha-ChainMolekülen in professionell antigenpräsentierenden Makrophagen und dendritischen Zellen, aber auch im Endothel, wie in $\bullet$ Abb. 3d gezeigt. Da sich in den In-situ-Hybridisierungen sowohl intakte als auch nekrobiotisch veränderte Myozyten sowie die epikardialen Blutgefäße als durchweg virusnegativ erwiesen, liegt die Vermutung nahe, dass sich die myokardialen Nekrosen sekundär im Rahmen einer ausgeprägten koronaren Mikrozirkulationsstörung mit B19-induzierter endothelialer Zytokinexpression erklären, wobei die Infektion bei einem Teil der Patienten mit den klinischen Zeichen eines Myokardinfarktes einhergeht. Im Gegensatz zur aku- ten B19-Infektion findet sich in der chronischen Phase der Erkrankung eine Viruspersistenz in Endothelien kleiner myokardialer Kapillaren mit makrophagenreicher Entzündung [14].

Im Gegensatz zur In-situ-Hybridisierung erfordert die PCR-Diagnostik der B19-Infektion an Endomyokardbiopsien höchste Ansprüche hinsichtlich der Kontrolle von Kontaminationen, die sich insbesondere durch eine hohe Viruslast von bis $10^{12}$ Viruspartikeln pro $\mathrm{ml}$ Serum bei akut infizierten Patienten erklären. Die Ergebnisse der "Nested-PCR-Analysen“ werden durch fluoreszenzgestützte quantitative PCR mit Bestimmung der Viruslast bestätigt. Diese liegt bei der akuten Infektion des Herzens zwischen $10^{4}$ und $10^{8}$ Genomäquivalenten pro $\mu \mathrm{g}$ isolierter Nukleinsäuren und bei entzündlicher B19-Persistenz zwischen $10^{2}$ und $10^{3}$ viralen Kopien. Die im Rahmen der Spezifitätskontrollen routinemäßig durchgeführten Mutationsanalysen viraler Genome begründen die Annahme, dass unterschiedliche B19-Genotypen und Virusvarianten die hochvariablen Verläufe hinsichtlich endothelialer und myokardialer Dysfunktion erklären könnten [3].

Eindeutige Hinweise für eine B19-assoziierte endotheliale Dysfunktion ergaben sich aus klinischen Beobachtungen [22] sowie aus funktionellen Studien zur Modulation der inflammatorisch wichtigen "mitogen-activated protein kinase“ (MAPK) und „Janus kinase (JAK)/Signal Transducers and Activators of Transcription" (STAT)-Signalwege, die u. a. für die Regulation der Expression von Zytokinen (Interferone und Interleukine) und Endothelfunktion verantwortlich sind [7]. Die Ergebnisse dieser Studien belegen, dass die B19-Infektion mit einer Aktivierung der „p44/42-extracellular signal-regulated kinase" (ERK), p38-MAPK und $\mathrm{STAT}_{3}$-Signalwege sowie der Expression des Negativregulators "protein inhibitor of activated $\mathrm{STAT}_{3}$ “ (PIAS 3 ) einhergeht

(• Abb.4). Eine initiale MAPK/STATAktivierung mit anschließender PIAS3Expression bewirkt eine Dysregulation des Interferonsystems und scheint mitverantwortlich für die Entwicklung einer chronischen B19-assoziierten inflammatorischen Kardiomyopathie (B19-iCMP) zu sein [7]. 
Die Infektion mit B19 kann zudem proapoptotische Prozesse in der Zielzelle auslösen, die bei der B19-iCMP zur Aktivierung/Dysfunktion der myokardialen Endothelzellen beitragen können [17]. Funktionelle Analysen zeigten die Aktivierung der Caspase 3 mit folgender „ $\mathrm{Na}^{+} /$ $\mathrm{H}^{+}$-exchanger 1-“ (NHE1-)Spaltung und DNA-Fragmentierung sowie Annexin-VBindung [17]. Die intrazelluläre $\mathrm{Ca}^{2+}-\mathrm{Ak}$ tivität ist ein wichtiger Regulator der Zellfunktion und spielt eine bedeutende Rolle bei Mechanismen, die zur endothelialen Dysfunktion führen. Ausgehend von dem kürzlich erfolgten Nachweis einer viralen PLA2-Aktivität in der VP1-Region von Parvoviren, konnte jetzt in funktionellen Experimenten die B19-VP1-PLA2-Aktivität hinsichtlich der zytosolischen $\mathrm{Ca}^{2+}$-Regulation belegt werden [17], ein Effekt der maßgeblich an der Pathophysiologie der endothelialen Dysfunktion beteiligt ist.

\section{Fazit für die Praxis}

\section{In der vorliegenden Übersicht wurde} aus kardiopathologischer Sicht dargelegt, dass die hohe Prävalenz viraler Genome von 46\% in Myokardbiopsien von Patienten mit klinisch vermuteter Myokarditis und dilatativer Kardiomyopathie ein Zusammenführen klinischer, infektiologischer, immunhistologischer und histomorphologischer Befunde erfordert, um eine pathogenetisch bedeutsame inflammatorische Infektion von einer harmlosen latenten myokardialen Viruspersistenz zu unterscheiden. Die kardiopathologische Diagnose einer virusassoziierten inflammatorischen Kardiomyopathie rechtfertigt vor dem Hintergrund der kardialen Beschwerdesymptomatik ein Umdenken hinsichtlich therapeutischer Konsequenzen. Eine immunsuppressive Therapie mit Kortikosteroiden ist generell nicht gerechtfertigt, da hierdurch eine erhöhte Virusreplikation und Hemmung des endogenen Interferonsystems induziert werden kann [11]. Eine immunsuppressive Behandlung der inflammatorischen Kardiomyopathie ist deshalb nur nach Ausschluss einer persistierenden Virusinfektion bei der postviralen Autoimmunmyokarditis gerechtfertigt. Eine antivirale Therapie mit menschlichen Immunglobulinen erscheint entsprechend den Leitli- nien der „Gesellschaft für Virologie“ v. a. bei der akuten Virusmyokarditis sinnvoll, die mit einer systemischen Infektion einhergeht. Kontrollierte randomisierte Therapiestudien stehen allerdings noch nicht zur Verfügung. Entsprechendes gilt für die antivirale Therapie mit Typ-1-Interferonen (IFN- $\alpha$, IFN- $\beta$ ), wobei eine Verbesserung hämodynamischer Parameter sowie eine mögliche Viruselimination aus dem Herzmuskel bislang nur durch Einzelfallstudien belegt ist. Neben der gegenwärtig in einer randomisierten Therapiestudie der Myokarditis im Kindesalter verfolgten IFN- $\alpha$-Therapie wird derzeit auch eine randomisierte multizentrische IFN- $\beta$-Therapiestudie bei viruspositiver inflammatorischer Kardiomyopathie im Erwachsenenalter durchgeführt [16]. Die entsprechenden Ergebnisse bleiben abzuwarten.

\section{Korrespondenzadresse}

\section{Prof. Dr. R. Kandolf}

Abteilung Molekulare Pathologie,

Universitätsklinikum Tübingen

Liebermeisterstraße 8, 72076 Tübingen

reinhard.kandolf@med.uni-tuebingen.de

Danksagung. Die dieser Übersicht zugrunde liegenden Forschungsarbeiten zur inflammatorischen Kardiomyopathie wurden mit Mitteln des Bundesministeriums für Bildung, Wissenschaft, Forschung und Technologie im Rahmen des „Interdisziplinären Zentrums für Klinische Forschung" (IZKF), Universitätsklinikum Tübingen (01KS9602) sowie im Nationalen Genomforschungsnetz (NGFN, Infektion und Entzündung, 01GS0114) gefördert. Die aktuellen Forschungsprojekte werden im Sonderforschungsbereich/Transregio 19 (SFB-TR 19, Teilprojekte B4 und B5) „Inflammatorische Kardiomyopathie“ (Berlin, Greifswald, Tübingen) durch die Deutsche Forschungsgemeinschaft unterstützt.

Interessenkonflikt. Der korrespondierende Autor gibt an, dass kein Interessenkonflikt besteht.

\section{Literatur}

1. Aretz HT (1987) Myocarditis: the dallas criteria. Hum Pathol 18: 619-624

2. Badorff C, Lee GH, Lamphear BJ et al. (1999) Enteroviral protease 2a cleaves dystrophin: evidence of cytoskeletal disruption in an acquired cardiomyopathy. Nat Med 5: 320-326

3. Bock CT, Klingel K, Aberle S et al. (2005) Human parvovirus b19: a new emerging pathogen of inflammatory cardiomyopathy. J Vet Med B Infect Dis Vet Public Health 52: 340-343

4. Brown KE, Anderson SM, Young NS (1993) Erythrocyte $p$ antigen: cellular receptor for b19 parvovirus. Science 262: 114-117

5. Brown KE, Hibbs JR, Gallinella G et al. (1994) Resistance to parvovirus b19 infection due to lack of virus receptor (erythrocyte $p$ antigen). N Engl J Med 330: 1192-1196
6. Bultmann BD, Klingel K, Sotlar K et al. (2003) Fatal parvovirus b19-associated myocarditis clinically mimicking ischemic heart disease: an endothelial cell-mediated disease. Hum Pathol 34: 92-95

7. Duechting A, Tschope C, Kaiser H et al. (2008) Human parvovirus b19 ns1 protein modulates inflammatory signaling by activation of stat3/pias 3 in human endothelial cells. J Virol (Epub ahead of print)

8. Grumbach IM, Heim A, Pring-Akerblom P et al. (1999) Adenoviruses and enteroviruses as pathogens in myocarditis and dilated cardiomyopathy. Acta Cardiol 54: 83-88

9. Huber M, Watson KA, Selinka HC et al. (1999) Cleavage of rasgap and phosphorylation of mitogenactivated protein kinase in the course of coxsackievirus b3 replication. J Virol 73: 3587-3594

10. Kandolf R (2004) Virus etiology of inflammatory cardiomyopathy. Dtsch Med Wochenschr 129: 2187-2192

11. Kandolf R, Marre R, Mertens T et al. (2000) Perikarditis und myokarditis; Klinische infektiologie. Urban \& Fischer, München Jena, S 338-348

12. Kandolf R, Sauter M, Aepinus C et al. (1999) Mechanisms and consequences of enterovirus persistence in cardiac myocytes and cells of the immune system. Virus Res 62: 149-158

13. Kandolf $R$ (2008) Klinische Kinderkardiologie. In: Schumacher G, Hess J, Bühlmeyer K (Hrsg) Klinische Kinderkardiolgoie, 4. Aufl. Springer, Berlin Heidelberg New York, S 449-460

14. Klingel K, Sauter M, Bock CT et al. (2004) Molecular pathology of inflammatory cardiomyopathy. Med Microbiol Immunol (Berl) 193: 101-107

15. Koch WC (2001) Fifth (human parvovirus) and sixth (herpesvirus 6) diseases. Curr Opin Infect Dis 14: 343-356

16. Kuhl U, Pauschinger M, BockT et al. (2003) Parvovirus b19 infection mimicking acute myocardial infarction. Circulation 108: 945-950

17. Lupescu A, Bock CT, Lang PA et al. (2006) Phospholipase a2 activity-dependent stimulation of ca2+ entry by human parvovirus b19 capsid protein vp1. J Virol 80: 11370-11380

18. McManus B, Kandolf R (2000) Atlas of cardiovascular pathology for the clinician. In: McManus B, Braunwald E (eds) Current Medicine. Philadelphia, pp 168-183

19. Pauschinger M, Bowles NE, Fuentes-Garcia FJ et al. (1999) Detection of adenoviral genome in the myocardium of adult patients with idiopathic left ventricular dysfunction. Circulation 99: 1348-1354

20. Richardson P, McKenna W, Bristow M et al. (1996) Report of the 1995 world health organization/international society and federation of cardiology task force on the definition and classification of cardiomyopathies. Circulation 93: 841-842

21. Selinka HC, Wolde A, Sauter M et al. (2004) Virusreceptor interactions of coxsackie $b$ viruses and their putative influence on cardiotropism. Med Microbiol Immunol (Berl) 193: 127-131

22. Tschope C, Bock CT, Kasner M et al. (2005) High prevalence of cardiac parvovirus b19 infection in patients with isolated left ventricular diastolic dysfunction. Circulation 111: 879-886

23. Weigel-Kelley KA, Yoder MC, Srivastava A (2003) Alpha5beta1 integrin as a cellular coreceptor for human parvovirus b19: requirement of functional activation of beta1 integrin for viral entry. Blood 102: 3927-3933

24. Wessely R, Klingel K, Santana LF et al. (1998) Transgenic expression of replication-restricted enteroviral genomes in heart muscle induces defective excitation-contraction coupling and dilated cardiomyopathy. J Clin Invest 102: 1444-1453 